\title{
Assessment of mitral regurgitation and mitral complex geometry in patients after transcatheter aortic valve implantation
}

\author{
Danuta Sorysz ${ }^{1}$, Agata Krawczyk-Ożóg ${ }^{1}$, Artur Dziewierz ${ }^{1,2}$, Tomasz Tokarek ${ }^{1}$, Barbara Zawiślak \\ Mateusz Hołda ${ }^{3,4}$, Kinga Komnata ${ }^{5}$, Andrzej Surdacki ${ }^{1,2}$, Stanisław Bartuś ${ }^{1,2}$, Dariusz Dudek ${ }^{1,2}$ \\ ${ }^{1}$ Department of Cardiology and Cardiovascular Interventions, University Hospital, Krakow, Poland \\ ${ }^{2}$ Second Department of Cardiology, Institute of Cardiology, Jagiellonian University Medical College, Krakow, Poland \\ ${ }^{3}$ HEART - Heart Embryology and Anatomy Research Team, Department of Anatomy, Jagiellonian University Medical College, Krakow, Poland \\ ${ }^{4}$ Division of Cardiovascular Sciences, The University of Manchester, Manchester, UK \\ ${ }^{5}$ Research Student, Jagiellonian University Medical College, Krakow, Poland
}

Adv Interv Cardiol 2020; 16, 3 (61): 300-305

DOI: https://doi.org/10.5114/aic.2020.99265

\begin{abstract}
A bstract
Introduction: Mitral regurgitation (MR) of varying degrees and mechanisms is a common finding in patients with aortic stenosis with different improvement after transcatheter aortic valve implantation (TAVI).

Aim: To evaluate the impact of TAVI on mitral complex geometry and the degree of MR.

Material and methods: A total of 31 patients (29.0\% males) with severe aortic stenosis and moderate or severe MR at the baseline who underwent TAVI were included in this study. Clinical and echocardiographic characteristics were determined at baseline and at 6 and 12 months.

Results: After TAVI, decrease of MR vena contracta width ( $p=0.00002, p=0.00004)$, aorto-mural mitral annulus diameter $(p=0.00008, p=0.02)$, increase of mitral annular plane systolic excursion $(p=0.0004, p=0.0003)$, left ventricular stroke volume $(p=0.0003, p=0.0004)$, ejection fraction $(p=0.0004, p=0.01)$ and decrease of major dimension of left ventricle in three chamber view $(p=0.05, p=0.002)$ were observed in patients at both time points. Additionally, we observed a decrease of distance between the head of the papillary muscles $(p=0.003)$ at 6 months and a decrease of left atrium volume index $(p=0.01)$ and systolic pulmonary artery pressure $(p=0.01)$ at 12 months.

Conclusions: Patients with moderate or severe MR undergoing TAVI achieved significant improvement of mitral valve complex function resulting in the reduction of MR degree.
\end{abstract}

Key words: transcatheter aortic valve implantation, mitral regurgitation, mitral valve complex, aortic stenosis.

Su m m a ry

In patients with severe aortic stenosis and moderate or severe mitral regurgitation undergoing a transcatheter aortic valve implantation procedure significant reductions of symptoms and improvement of mitral valve complex function resulting in the reduction of mitral regurgitation degree are observed, as well as a significant improvement of the left ventricle geometry and functions.

\section{Introduction}

The mitral valve apparatus is a complex consisting of the annulus, leaflets, commissures, tendinous cords, papillary muscles and the left atrial and ventricular walls $[1,2]$. Assessment of all components of the mitral apparatus is necessary to determine the mechanism of its incompetency. Mitral regurgitation (MR) can be classified as primary (due to structural abnormalities of the valve), secondary (functional), associated with left ventricle dysfunction (ischemic and non-ischemic MR) and as a consequence of atrial fibrillation (AF).

$M R$ is frequently present in patients with severe aortic stenosis (AS) [3]. Mitral valve deformation and

\section{Corresponding author:}

Danuta Sorysz MD, PhD, Department of Cardiology and Cardiovascular Interventions, University Hospital, 2 Jakubowskiego St, $30-688$ Krakow, Poland, phone: +48 1240022 50, e-mail: dtsorysz@op.pl

Received: 2.06.2020, accepted: 9.06.2020. 
tethering, as well as an increase in transmitral pressure gradient caused by AS and increased left ventricle (LV) pressure, all contribute to MR [4]. Additionally, with increasing age, the incidence of AS and coronary artery disease may lead to development of functional ischemic MR $[5,6]$. In the PARTNER trial, the incidence of concomitant moderate-to-severe $M R$ in patients with severe AS was approximately $20 \%[7,8]$. However, the necessity of intervention for both valves may increase the risk of operation to an unacceptable level, especially in high-risk patients. Transcatheter aortic valve implantation (TAVI) is a feasible option for the treatment of severe AS in patients at high risk of conventional surgical aortic valve replacement [9-11].

\section{Aim}

In this study, we sought to assess the impact of TAVI on geometry and function of the mitral apparatus in patients with at least a moderate degree of $M R$ in the pre-procedural transthoracic echocardiography (TTE). Detailed aims of this study were: 1) to characterize the degree of MR by TTE at 6- and 12-month follow-up after TAVI in patients with moderate or severe MR before TAVI; 2) to investigate the impact of TAVI on TTE parameters assessing geometry and function of the mitral valve complex: mitral annulus, left atrium and left ventricle.

\section{Material and methods}

\section{Study population}

This study was approved by the Bioethical Committee of the Jagiellonian University, Cracow, Poland. The study protocol conforms to the ethical guidelines of the 1975 Declaration of Helsinki. All patients provided informed consent for participation in the study. We analyzed all patients with severe AS admitted to the Department of Cardiology and Cardiovascular Interventions, University Hospital in Krakow between January 2015 and December 2016. We included 31 patients $(29.0 \%$ males, $82.1 \pm 5.2$ years old) with severe AS and concomitant moderate or severe $M R$, recognized during TTE according to the recommendations for the echocardiographic assessment of native valvular regurgitation [12]. Patients without a sufficient acoustic window were excluded. All patients had high risk or serious contraindications for surgical aortic valve replacement and were qualified for TAVI by a multidisciplinary "Heart Team". Clinical and TTE follow-up was conducted through control visits of the patient at 6 and 12 months after TAVI (Edwards Sapien XT valve; Edwards Lifesciences, Irvine, CA, USA, Evolut R Medtronic Scientific, Minneapolis, MN, USA).

\section{Echocardiographic assessment}

All patients underwent TTE, including M-mode, two-dimensional, three-dimensional, and Doppler im- aging, at baseline and during follow-up. Close attention was paid to all acquisition settings in order to maximize image quality. For better visualization of mitral and aortic valve anatomy and function, two-dimensional and three-dimensional transesophageal echocardiography (TEE) was performed at baseline. All TTE and TEE examinations were performed using Vivid E9 (GE Healthcare, Waukesha, WI, USA). The post-processing evaluation was performed using a dedicated workstation (EchoPAC, GE Healthcare, Waukesha, WI, USA). The linear measurements were taken using virtual calipers. The measurements were performed according to the current recommendations $[12,13]$.

The following qualitative and quantitative echocardiography parameters were performed to assess the severity of MR: vena contracta (VC) width (in the case of multiple jets: vena contracta of the dominant jet) and peak E-wave; to assess mitral valve geometry: mitral annulus diameters at end-diastole: aorto-mural diameter in parasternal long-axis TTE view and intercommissural diameter in the modified apical two-chamber view; aorto-mural annulus/anterior leaflet ratio; maximal and mean transvalvular mitral gradient; maximal and mean transvalvular aortic gradient; peak aortic valve velocity (Vmax); and aortic valve area (AVA). The antero-mural diameter of the mitral annulus $>35 \mathrm{~mm}$ or the aorto-mural annulus/anterior leaflet ratio $>1.3$ was considered to indicate mitral annular dilatation $[12,14]$.

Additionally, particular left ventricle geometry and function parameters were evaluated: end-diastolic and end-systolic left ventricle diameters in parasternal long-axis; left ventricle sphericity index in apical four-chamber view; major left ventricle dimension in three-chamber view; distance between heads of left ventricular papillary muscles in end-diastolic phase; left ventricle ejection fraction; stroke volume; and mitral annular plane systolic excursion. Other parameters include left atrium diameter in parasternal long-axis view; left atrium indexed volume; right atrium area and indexed volume; right ventricle linear dimension (maximal transversal dimension in the basal one third of right ventricle inflow at end-diastole); systolic pulmonary artery pressure; and grade of tricuspid regurgitation.

\section{Statistical analysis}

Standard descriptive statistical methods were used. The data were presented as mean values with their corresponding standard deviations. The normality of the data was assessed with the Shapiro-Wilk test. One-way analysis with the unpaired two-sample $T$-test (for normal distribution) or the Mann-Whitney $U$ test (for non-normally distributed data) was applied for quantitative variables. The analysis of variance (ANOVA) or non-parametric Kruskal-Wallis test was used to compare values between different groups. Detailed comparisons were 
performed using Tukey's post hoc analyses. Qualitative variables were compared using the $\chi^{2}$ (chi-squared) test of proportions with Bonferroni correction to account for multiple comparisons. We performed statistical analyses with Statisticav13 (StatSoft Inc., Tulsa, OK, USA). The statistical significance was set at a $p$-value lower than 0.05 .

\section{Results}

The baseline clinical characteristics of patients are shown in Table I. At baseline, patients presented heart failure symptoms that were mainly $(58.1 \%)$ in class III of the New York Heart Association (NYHA) Functional Classification (Table II). Moderate MR was diagnosed in $93.5 \%$ of patients and severe in $6.5 \%$. Calcifications in the mitral valve annulus were found in $61.3 \%$ of patients and in mitral valve leaflets in $22.6 \%$ of patients. Annular mitral dilatation was identified in $64.5 \%$ of patients. MR was accompanied by moderate and severe TR in $41.9 \%$ and $9.7 \%$ of patients, respectively.

One patient died 2 months after TAVI and therefore the follow-up group at 6 and 12 months consisted of 30 patients. Table II presents clinical and echocardiographic data at baseline and during follow-up. A significant decrease of heart failure symptoms assessed with NYHA class was observed both 6 and 12 months after TAVI as compared to baseline (Table II; $p<0.05$ ). Several critical MR parameters showed significant improvement

Table I. Baseline clinical characteristics of patients

\begin{tabular}{|c|c|}
\hline Variable & $\begin{array}{c}\text { Number (\%) } \\
\text { or mean } \pm \text { SD }\end{array}$ \\
\hline Age, mean \pm SD [years] & $82.1 \pm 5.2$ \\
\hline $\begin{array}{l}\text { Coronary artery disease confirmed } \\
\text { in angiography, } n(\%)\end{array}$ & $20(64.5)$ \\
\hline Previous myocardial infarction, $n$ (\%) & $9(29.0)$ \\
\hline $\begin{array}{l}\text { Previous percutaneous coronary intervention, } \\
n(\%)\end{array}$ & $14(45.2)$ \\
\hline Previous coronary artery bypass grafting, $n(\%)$ & $4(12.9)$ \\
\hline Cardiac resynchronization therapy, $n$ (\%) & 0 \\
\hline Pacemaker, $n$ (\%) & $7(22.6)$ \\
\hline Arterial hypertension, $n(\%)$ & $28(90.3)$ \\
\hline Diabetes mellitus type II, $n$ (\%) & $9(29.0)$ \\
\hline Atrial fibrillation, $n(\%)$ & $20(64.5)$ \\
\hline Hyperlipidemia, $n$ (\%) & $12(38.7)$ \\
\hline Ever-smoker, $n(\%)$ & $2(6.5)$ \\
\hline GFR $\left[\mathrm{ml} / \mathrm{min} / 1.73 \mathrm{~m}^{2}\right]$ & $55.4 \pm 15.4$ \\
\hline Previous stroke/transient ischemic attack, $n$ (\%) & $1(9.7)$ \\
\hline Body mass index, mean $\pm \mathrm{SD}\left[\mathrm{kg} / \mathrm{m}^{2}\right]$ & $26.1 \pm 3.7$ \\
\hline Body surface area, mean \pm SD $\left[\mathrm{m}^{2}\right]$ & $1.7 \pm 0.2$ \\
\hline
\end{tabular}

SD - standard deviation. after the procedure, which included a decrease in VC (baseline: $5.9 \pm 1.5 \mathrm{~mm}$ vs. 6 months: $3.8 \pm 1.6 \mathrm{~mm}$ vs. 12 months: $3.2 \pm 1.4 \mathrm{~mm}$, Table II, $p<0.001$ ).

All patients in the studied group achieved a significant increase of AVA ( $p=0.000006, p=0.00002)$ and a decrease of maximal ( $p=0.000003, p=0.000008)$ and mean ( $p=0.000003, p=0.000008)$ transvalvular aortic gradient. After TAVI, decrease of MR, especially VC width ( $p=0.00002, p=0.00004)$ and aorto-mural mitral annulus diameter $(p=0.00008, p=0.02$ ), was achieved. Additionally, an increase of mitral annular plane systolic excursion ( $p=0.0004, p=0.0003)$, left ventricular stroke volume (LVSV) $(p=0.0003, p=0.0004)$ and LVEF $(p=0.0004, p=0.01)$ and a decrease of major dimension of the left ventricle in three-chamber view $(p=0.05$, $p=0.002)$ and left ventricular end-systolic diameter (LVESD) ( $p=0.004, p=0.02$ ) were observed in patients at both time points. We observed a decrease of distance between the head of the papillary muscles $(p=0.003)$ at 6 months and a decrease of left atrium volume index (LAVi) $(p=0.01)$ and systolic pulmonary artery pressure (sPAP) $(p=0.01)$ at 12 months. Detailed TTE data are listed in Table II.

\section{Discussion}

Mitral regurgitation in patients with severe SA referred for TAVI may have functional, organic, or complex etiology. The latter is most frequent in the group of elderly patients. Degeneration and calcifications of the valve are frequent and usually affect the leaflets, tendinous cord and annulus [15]. In the studied group, calcifications of the mitral annulus dominated (61.3\%). On the other hand, coronary heart disease is common in patients after TAVI, including in the form of previous myocardial infarction, which may be the cause of left ventricular remodeling and ischemic MR. Additionally, severe SA with elevated end-systolic LV pressure may aggravate subendocardial ischemia with deterioration of the left ventricle function and cause changes of geometry which may also be a component of non-ischemic functional MR.

Both mentioned components of functional MR led to mitral apparatus geometry deformation, including leaflet restriction, enlargement of the mitral ring, and increase in distance between papillary muscles. Considering the complexity of the MR pathomechanism in patients with AS and improvement of left ventricular function after $T A V I$, a reduction in the significance of MR after the procedure should be expected [16].

There has been much discussion regarding the reduction of MR after isolated aortic valve replacement. Following surgical aortic valve replacement, improvement in MR was reported in $27-82 \%$ of the patients [17]. Webb et al. reported an improvement in MR in 24 out of the 50 patients (48\%) with moderate to severe MR following the implantation of the Edwards Sapien Valve [18]. In another 
study in 171 patients with MR grade $\geq 2,29 \%$ improved to $M R$ grade $\leq 1$. TAVI had no influence on MR grade at follow-up [19]. Functional MR, coronary artery disease and absence of atrial fibrillation have been identified as predictors of reduction of MR after TAVI [20]. Our study shows that TAVI improves the grade of MR (Figure 1) at 6 and 12 months, which could be a result of improvement in geometry of the mitral complex and left ventricle function. Only 9 (30\%) patients did not achieve a reduction of MR grade at any analyzed time points. A significant reduction

Table II. Results of obtained echocardiographic data (meantstandard deviation) at baseline and during follow-up

\begin{tabular}{|c|c|c|c|c|c|}
\hline Parameter & Baseline & 6 months & $\begin{array}{c}P \text {-value } \\
\text { baseline vs. } \\
6 \text { months }\end{array}$ & 12 months & $\begin{array}{c}P \text {-value } \\
\text { baseline vs. } \\
12 \text { months }\end{array}$ \\
\hline \multicolumn{6}{|l|}{ Mitral regurgitation, $n(\%)$ : } \\
\hline Trivial & 0 & 2 & \multirow[t]{4}{*}{$0.01^{*}$} & 3 & \multirow[t]{4}{*}{0.054} \\
\hline Mild & 0 & 16 & & 16 & \\
\hline Moderate & 29 & 12 & & 11 & \\
\hline Severe & 1 & 0 & & 0 & \\
\hline $\mathrm{VC}[\mathrm{mm}]$ & $5.9 \pm 1.5$ & $3.8 \pm 1.6$ & $0.00002^{*}$ & $3.2 \pm 1.4$ & $0.00004^{*}$ \\
\hline$E[\mathrm{~m} / \mathrm{s}]$ & $1.0 \pm 0.2$ & $1.0 \pm 0.3$ & 0.4 & $1.0 \pm 0.2$ & 0.6 \\
\hline Intercommissural mitral annulus diameter [mm] & $31.7 \pm 4.4$ & $30.0 \pm 4.5$ & $0.01^{*}$ & $30.7 \pm 4.7$ & 0.1 \\
\hline Aorto-mural mitral annulus diameter [mm] & $29.0 \pm 5.6$ & $27.1 \pm 5.2$ & $0.00008^{*}$ & $27.7 \pm 5.1$ & $0.02^{*}$ \\
\hline Aorto-mural mitral annulus/anterior leaflet ratio & $1.5 \pm 0.3$ & $1.4 \pm 0.31$ & 0.4 & $1.4 \pm 0.29$ & $0.03^{*}$ \\
\hline $\mathrm{PGmv}[\mathrm{mm} \mathrm{Hg}]$ & $6.3 \pm 2.0$ & $8.3 \pm 4.4$ & $0.08^{*}$ & $7.2 \pm 3.6$ & 0.2 \\
\hline MPGmv [mm Hg] & $2.8 \pm 1.0$ & $3.3 \pm 1.6$ & 0.1 & $2.6 \pm 1.3$ & 0.6 \\
\hline PGao [mm Hg] & $82.2 \pm 36.8$ & $23.2 \pm 10.9$ & $0.000003^{*}$ & $23.9 \pm 10.3$ & $0.000008^{*}$ \\
\hline MPGao [mm Hg] & $50.9 \pm 23.5$ & $12.5 \pm 6.2$ & $0.000003^{*}$ & $13.8 \pm 6.5$ & $0.000008^{*}$ \\
\hline Vmax & $4.4 \pm 1.0$ & $2.3 \pm 0.5$ & $0.000003^{*}$ & $2.4 \pm 0.5$ & $0.000006^{*}$ \\
\hline AVA $\left[\mathrm{cm}^{2}\right]$ & $0.58 \pm 0.2$ & $1.5 \pm 0.3$ & $0.000006^{*}$ & $1.4 \pm 0.3$ & $0.00002^{*}$ \\
\hline LVEDD [mm] & $48.9 \pm 13.6$ & $47.1 \pm 9.5$ & 0.3 & $46.5 \pm 7.7$ & 0.02 \\
\hline LVESD [mm] & $36.7 \pm 12.4$ & $31.8 \pm 8.5$ & $0.004^{*}$ & $32.7 \pm 8.9$ & $0.02^{*}$ \\
\hline LVSV [ml] & $61.3 \pm 15.0$ & $75.3 \pm 18.8$ & $0.0003^{*}$ & $70.8 \pm 13.6$ & $0.004^{*}$ \\
\hline $\mathrm{SFi}$ & $0.62 \pm 0.086$ & $0.59 \pm 0.089$ & 0.6 & $0.60 \pm 0.086$ & 0.5 \\
\hline Major dimension of LV in three-chamber view [mm] & $77.9 \pm 10.9$ & $76.3 \pm 9.6$ & $0.047^{\star}$ & $72.8 \pm 8.2$ & $0.002^{*}$ \\
\hline Distance between head of papillary muscle & $23.5 \pm 6.0$ & $20.7 \pm 5.1$ & $0.003^{*}$ & $21.0 \pm 7.0$ & 0.1 \\
\hline $\operatorname{LVEF}(\%)$ & $51.6 \pm 17.1$ & $59.2 \pm 13.3$ & $0.0004^{*}$ & $59.5 \pm 13.3$ & $0.01^{*}$ \\
\hline LAd $[\mathrm{cm}]$ & $4.8 \pm 0.6$ & $4.9 \pm 0.7$ & 0.8 & $4.8 \pm 0.6$ & 0.6 \\
\hline LAVi $\left[\mathrm{ml} / \mathrm{m}^{2}\right]$ & $74.8 \pm 19.0$ & $68.7 \pm 18.8$ & 0.2 & $62.9 \pm 18.5$ & $0.01^{*}$ \\
\hline $\operatorname{RAA}\left[\mathrm{cm}^{2}\right]$ & $18.0 \pm 8.0$ & $19.8 \pm 6.8$ & 0.99 & $19.6 \pm 5.7$ & 0.9 \\
\hline $\operatorname{RAVi}\left[\mathrm{ml} / \mathrm{m}^{2}\right]$ & $38.5 \pm 16.2$ & $38.2 \pm 20.8$ & 0.7 & $35.3 \pm 14.3$ & 0.3 \\
\hline RV dimension [mm] & $37.1 \pm 6.3$ & $33.4 \pm 9.6$ & 0.125 & $36.8 \pm 5.4$ & 0.95 \\
\hline $\mathrm{sPAP}[\mathrm{mm} \mathrm{Hg}]$ & $52.6 \pm 11.9$ & $52.3 \pm 15.6$ & 0.97 & $45.2 \pm 11.1$ & $0.01^{*}$ \\
\hline MAPSE [mm] & $11.1 \pm 2.5$ & $13.1 \pm 2.6$ & $0.0004^{*}$ & $13.6 \pm 2.4$ & $0.0003^{*}$ \\
\hline \multicolumn{6}{|l|}{ Tricuspid regurgitation: } \\
\hline Trivial & 2 & 6 & \multirow[t]{4}{*}{0.3} & 8 & \multirow[t]{4}{*}{0.07} \\
\hline Mild & 12 & 14 & & 11 & \\
\hline Moderate & 12 & 7 & & 8 & \\
\hline Severe & 3 & 3 & & 3 & \\
\hline \multicolumn{6}{|l|}{ NYHA: } \\
\hline 1 & 0 & 7 & \multirow[t]{4}{*}{$0.0002^{*}$} & 8 & \multirow[t]{4}{*}{$0.002^{*}$} \\
\hline II & 9 & 19 & & 19 & \\
\hline III & 18 & 4 & & 3 & \\
\hline IV & 1 & 0 & & 0 & \\
\hline
\end{tabular}

$N$ - number of samples, VC - vena contracta width $L V$ - left ventricle, $R V$ - right ventricle, LA - left atrium, RA - right atrium, PGmv - maximal transvalvular mitral gradient, MPGmv - mean transvalvular mitral gradient, $P G a o-$ maximal transvalvular aortic gradient, MPGao - mean transvalvular aortic gradient, Vmax - peak aortic valve velocity, LVEDD - end-diastolic LV diameter, LVESD - left ventricular end-systolic diameter, LVSV - left ventricular stroke volume, SFi - LV sphericity index, LVEF - LV ejection fraction, LAd - left atrium diameter, LAVi - left atrium volume index, RAA - right atrium area, RAVi - right atrium volume index, SPAP - systolic pulmonary artery pressure, MAPSE - mitral annular plane systolic excursion, *statistically significant. 

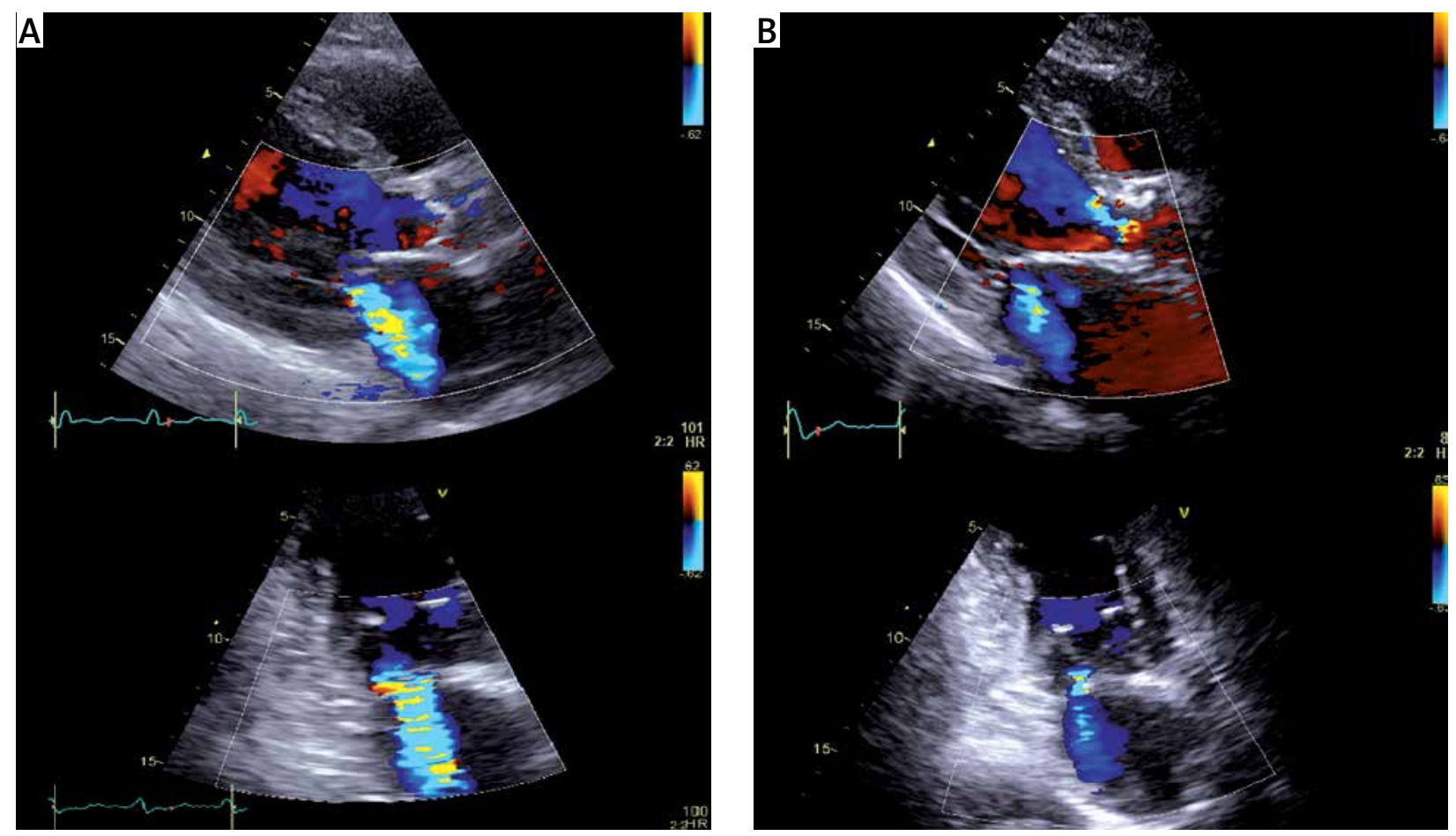

Figure 1. Color flow mitral regurgitation jet in transthoracic echocardiography before (A) and after (B) transcatheter aortic valve implantation in the same patient $L A$ - left atrium, $L V$ - left ventricle.

of the diameter of the mitral annulus and distance between the heads of the papillary muscles was observed. The detailed study of Tayyareci et al. also showed the importance of mitral annulus diameter and area reduction (2D and 3D analysis) in the group with MR improvement. Moreover, they observed no effect of TAVI on MR in the group with restriction of mitral leaflets, which is a consequence of mitral complex geometry change, as the greater distance between papillary muscles [16]. In our study, a significant decrease of sPAP was observed, although it may be the result of aortic valve implantation as well as a decrease in the significance of MR. This was reflected in a significant improvement in exercise capacity (NYHA class).

As coronary artery disease was present in two-thirds of patients and one-third of patients had a history of myocardial infarction, the functional component of the MR etiology seems to be the most frequent in the studied group. A significant reduction in left ventricular diameter and an improvement in left ventricular systolic function were observed, reflected in an increase in LVEF and mitral annular plane systolic excursion (MAPSE). Interestingly, the reduction in end-diastolic dimension achieves statistical significance 1 year after the procedure, in contrast to the end-diastolic dimension and ejection fraction. Relatively immediate increase in ejection fraction observed as early as 6 months after TAVI may be the result of a significant decrease in afterload and transvalvular gradients after TAVI valve implantation. In contrast, the process of reversing negative remodeling is long-lasting, and hence the reduction in end-diastolic volume was confirmed only in an annual follow-up. The observed increase in LVSV may be caused by both an increase in LVEF and a reduction in the significance of MR.

The present study has two major limitations. The most important one is its single-center character and the relatively small sample size. To eliminate these disadvantages, further, large, multicenter clinical studies with extended follow-up are required to confirm the findings of our study.

\section{Conclusions}

Patients with severe AS and moderate or severe MR undergoing TAVI achieved a significant reduction of symptoms and improvement of mitral valve complex function resulting in the reduction of MR degree. Furthermore, a significant improvement in the left ventricle geometry and function was achieved.

\section{Conflict of interest}

The authors declare no conflict of interest.

\section{References}

1. Krawczyk-Ozóg A, Hołda MK, Sorysz D, et al. Morphologic variability of the mitral valve leaflets. J Thorac Cardiovasc Surg 2017; 154: 1927-35.

2. Krawczyk-Ożóg A, Hołda MK, Bolechała F, et al. Anatomy of the mitral sub-valvular apparatus. J Thorac Cardiovasc Surg 2017; 154: 1927-35. 
3. Baumgartner H, Falk V, Bax JJ, et al. 2017 ESC/EACTS Guidelines for the management of valvular heart disease. Eur Heart J 2017; 38: 2739-86.

4. Lindroos M, Kupari M, Heikkilä J, et al. Prevalence of aortic valve abnormalities in the elderly: an echocardiographic study of a random population sample. J Am Coll Cardiol 1993; 21: 1220-5.

5. Unger P, Clavel MA, Lindman BR, et al. Pathophysiology and management of multivalvular disease. Nat Rev Cardiol 2016; 13: 429-40.

6. Nombela-Franco L, Ribeiro HB, Urena M, et al. Significant mitral regurgitation left untreated at the time of aortic valve replacement: a comprehensive review of a frequent entity in the transcatheter aortic valve replacement era. J Am Coll Cardiol 2014; 63: 2643-58.

7. Kodali SK, Moses JW. Coronary artery disease and aortic stenosis in the transcatheter aortic valve replacement era: old questions, new paradigms: the evolving role of percutaneous coronary intervention in the treatment of patients with aortic stenosis. Circulation 2012; 125: 975-7.

8. Smith CR, Leon MB, Mack MJ, et al. Transcatheter versus surgical aortic-valve replacement in high-risk patients. N Engl I Med 2011; 364: 2187-98.

9. Leon MB, Smith CR, Mack M, et al. Transcatheter aortic-valve implantation for aortic stenosis in patients who cannot undergo surgery. N Engl J Med 2010; 363: 1597-607.

10. Kodali SK, Williams MR, Smith CR, et al. Two-year outcomes after transcatheter or surgical aortic-valve replacement. $\mathrm{N}$ Engl J Med 2012; 366: 1686-95.

11. Mack MJ, Leon MB, Smith CR, et al. 5-year outcomes of transcatheter aortic valve replacement or surgical aortic valve replacement for high surgical risk patients with aortic stenosis (PARTNER 1): a randomised controlled trial. Lancet 2015; 385: 2477-84.

12. Lancellotti P, Tribouilloy C, Hagendorff A, et al. Recommendations for the echocardiographic assessment of native valvular regurgitation: an executive summary from the European Association of Cardiovascular Imaging. Eur Heart J Cardiovasc Imaging 2013; 14: 611-44.

13. Lang RM, Badano LP, Mor-Avi V, et al. Recommendations for cardiac chamber quantification by echocardiography in adults: an update from the American society of echocardiography and the European association of cardiovascular imaging. Eur Heart J Cardiovasc Imaging 2015; 16: 233-71.

14. Caldarera I, Van Herwerden LA, Taams MA, et al. Multiplane transoesophageal echocardiography and morphology of regurgitant mitral valves in surgical repair. Eur Heart J 1995; 16: 999-1006.

15. Antoine C, Mantovani F, Benfari G, et al. Pathophysiology of degenerative mitral regurgitation. Circ Cardiovasc Imaging 2018; 11: e005971.

16. Tayyareci Y, Dworakowski R, Kogoj P, et al. Impact of mitral geometry and global afterload on improvement of mitral regurgitation after trans-catheter aortic valve implantation. Echo Res Pract 2016; 3: 71-8.

17. Tzikas A, Piazza N, Van Dalen BM, et al. Changes in mitral regurgitation after transcatheter aortic valve implantation. Catheter Cardiovasc Interv 2010; 75: 43-9.

18. Webb JG, Pasupati S, Humphries K, et al. Percutaneous transarterial aortic valve replacement in selected high-risk patients with aortic stenosis. Circulation 2007; 116: 755-63.

19. Boerlage-Van Dijk K, Wiegerinck EMA, Takama T, et al. Mitral regurgitation prior to transcatheter aortic valve implantation influences survival but not symptoms. Int J Cardiol 2016; 204: 95-100.

20. Muratori M, Fusini L, Tamborini G, et al. Mitral valve regurgitation in patients undergoing TAVI: impact of severity and etiology on clinical outcome. Int J Cardiol 2020; 299: 228-34. 